\title{
Article \\ Risk of Heart Failure in Patients with ST-Elevation Myocardial Infarction Receiving Drug-Eluting Stent Implantation and Undefined Duration of Antiplatelets
}

\author{
Li-Nien Chien ${ }^{1,2}{ }^{\mathbb{D}}$, Chun-Chao Chen ${ }^{3}$, Ya-Hui Chang ${ }^{4,5}$, Fa-Chang Yu ${ }^{6}$, Chen-Ting Tsai ${ }^{6}$, Hung-Yi Liu ${ }^{1}$, \\ Hung-I Yeh ${ }^{4,6}$ (D) and Chao-Feng Lin ${ }^{4,6,7, *}$
}

\section{check for}

updates

Citation: Chien, L.-N.; Chen, C.-C.; Chang, Y.-H.; Yu, F.-C.; Tsai, C.-T.; Liu, H.-Y.; Yeh, H.-I.; Lin, C.-F. Risk of Heart Failure in Patients with ST-Elevation Myocardial Infarction Receiving Drug-Eluting Stent Implantation and Undefined Duration of Antiplatelets. J. Pers. Med. 2022, 12, 369. https://doi.org/ 10.3390/jpm12030369

Academic Editor: Chia-Ti Tsai

Received: 12 January 2022

Accepted: 25 February 2022

Published: 28 February 2022

Publisher's Note: MDPI stays neutral with regard to jurisdictional claims in published maps and institutional affiliations.

Copyright: (C) 2022 by the authors. Licensee MDPI, Basel, Switzerland. This article is an open access article distributed under the terms and conditions of the Creative Commons Attribution (CC BY) license (https:// creativecommons.org/licenses/by/ $4.0 /)$.
1 School of Health Care Administration, College of Management, Taipei Medical University, Taipei 110, Taiwan; lnchien@tmu.edu.tw (L.-N.C.); dieseabook@gmail.com (H.-Y.L.)

2 Health Data Analytics and Statistics Center, Office of Data Science, Taipei Medical University, Taipei 110, Taiwan

3 Division of Cardiology, Department of Internal Medicine, Shuang Ho Hospital, Taipei Medical University, New Taipei City 235, Taiwan; b101092035@tmu.edu.tw

4 Department of Medicine, MacKay Medical College, New Taipei City 252, Taiwan; yahui057@gamil.com (Y.-H.C.); hungi.yeh@msa.hinet.net (H.-I.Y.)

5 Department of Pharmacy, MacKay Memorial Hospital, Taipei 104, Taiwan

6 Division of Cardiology, Department of Internal Medicine, MacKay Memorial Hospital, Taipei 104, Taiwan; buebueman0829@gmail.com (F.-C.Y.); chengtingtsai@gmail.com (C.-T.T.)

7 Ph.D. Program for Cancer Molecular Biology and Drug Discovery, College of Medical Science and Technology, Taipei Medical University and Academia Sinica, Taipei 115, Taiwan

* Correspondence: thcpci@gmail.com

\begin{abstract}
It remains unknown as to whether the use of new-generation drug-eluting stent (NG-DES) in patients with ST-elevation myocardial infarction (STEMI) who receive an undefined duration of dual antiplatelet therapy (DAPT) reduces the risk of hospitalization for heart failure (HHF). In this population-based retrospective cohort study, we applied propensity score matching to select 6831 pairs of patients with STEMI who had similar baseline characteristics and received either NGDES or bare-metal stent (BMS) implantation between 1 January 2007 and 31 December 2016. The risk of stent-associated HHF was evaluated, wherein death was considered a competing risk. Rates of cumulative incidence competing risk for $\mathrm{HHF}$ at the 1,2, 3, 4, and 5 year follow-up were lower in the NG-DES group $(3.79 \%, 5.21 \%, 6.15 \%, 7.01 \%$, and $8.29 \%$, respectively) than in the BMS group $(4.51 \%, 6.21 \%, 7.32 \%, 8.33 \%$, and $9.83 \%$, respectively). NG-DES implantation was associated with a lower risk of HHF than BMS implantation after 5 years, with an adjusted subdistribution hazard ratio of $0.82(95 \%$ confidence interval $0.72-0.92, p=0.001)$. These results accord with those of patients who received DAPT for $>6$ months. Our findings highlight that NG-DESs may reduce HHF risk in patients with STEMI receiving an undefined duration of DAPT.
\end{abstract}

Keywords: heart failure; ST-elevation myocardial infarction; drug-eluting stent; bare-metal stent

\section{Introduction}

Heart failure (HF) after myocardial infarction (MI) is a strong predictor for all-cause death in patients with MI [1]. In a population-based cohort study [1], including a mean follow-up duration of 7.6 years, the mortality rates in patients who developed post-MI HF were $70 \%$, whereas those in patients who did not develop post-MI HF were $28 \%$. In addition, patients who had HF after discharge from the index MI admission had a worse outcome than did those patients who had HF during MI admission [1], indicating that a prevention strategy is required to reduce the long-term risk of HF after MI.

A 12 month duration of dual antiplatelet therapy (DAPT) is recommended in patients with ST-elevation MI (STEMI), while a short DAPT duration of 6 months is recommended 
in those patients who have features of high bleeding risk (HBR) [2,3]. In addition, the use of new-generation drug-eluting stent (NG-DES) in patients with STEMI has demonstrated superior benefits compared with that of bare-metal stent (BMS) in terms of target vessel failure (TVF) and repeat percutaneous coronary intervention (PCI) [2], all according to randomized controlled trial (RCT) [4-6] and meta-analysis [7,8] results. However, the aforementioned studies only investigated specific NG-DES types and did not measure the risk of new-onset HF after stent implantation as the principal study outcome [4-8]. In addition, the adherence of DAPT at 12 month follow-up in patients in the previous studies [4-8] was $>90 \%$, implying that the results could not be generalized to the patients suspected to have HBR along with uncertain DAPT compliance. Nonadherence to DAPT undoubtedly put patients at high risk of recurrent ischemic events, which potentially leads to the development of HF. Hence, in real clinical situations, it remains poorly understood as to whether the use of NG-DES, compared with that of BMS, is associated with a reduced long-term risk of HF after stent implantation in patients with STEMI who received undefined duration of DAPT.

The comparison between NG-DES and BMS in patients with STEMI is hardly addressed by RCTs because the use of NG-DES has become the recommended treatment strategy [2]. In Taiwan, the cost of NG-DES is paid by patients, whereas the cost of BMS is fully covered by the National Health Insurance (NHI). Although the cost of stents may influence the choice of the type of stent used, it provided an opportunity to compare the risk of stent-associated HF between NG-DES and BMS in patients with STEMI. Therefore, the current study investigated the effects of NG-DES implantation on the risk of hospitalization for HF (HHF) in patients with STEMI who received undefined duration of DAPT at the 5 year follow-up by using a real-world data from Taiwan's NHI Research Database (NHIRD).

\section{Materials and Methods}

\subsection{Data Source}

This study was approved by the Institutional Review Board of MacKay Memorial Hospital (MMH-IRB; approval no. 20MMHIS351e). NHIRD is a database that contains the claims data of $99 \%$ of Taiwan's residents covered by NHI. Because individual identifiers in the NHIRD have been encrypted and cannot be recognized, the need for informed consent was waived under the full review process of the MMH-IRB. The NHIRD includes data on inpatient, outpatient, and prescription drug claims. The prescribed medications can be classified using the Anatomical Therapeutic Chemical (ATC) system; disease diagnoses are coded according to the International Classification of Diseases, Ninth Revision, Clinical Modification (ICD-9-CM) until 2016 and according to the International Classification of Diseases, Tenth Revision (ICD-10), subsequently. Death records from the National Death Registry are linked to the NHIRD on the basis of patients' encrypted identifiers [9].

\subsection{Study Cohort}

In the retrospective cohort, we included patients who were admitted for a primary diagnosis of MI (ICD-9-CM 410 and ICD-10 I21.0-I21.4, I22.0-I22.2, I22.8, and I22.9) between 2007 and 2016. The date of the first MI admission was considered the index date of MI. We excluded patients who were aged $<20$ years, were not Taiwanese citizens, had died during admission, had a previous MI or HF diagnosis, had received ivabradine or angiotensin receptor/neprilysin inhibitor (the recommended medications for chronic $\mathrm{HF}$ with systolic dysfunction) [10], had received PCI before admission, did not receive PCI or stent implantation, or had received polymer-free drug-coating stent or combined DES and BMS implantation at index MI admission. We also excluded patients who received first-generation DES implantation, which is associated with an increased stent thrombosis risk [11], and in turn, raises safety concerns and leads to a considerably decreased use in Taiwan. Moreover, patients with high HF risk, including those who received coronary artery bypass grafting [12], ventricular assist device support [13], valvular surgery [14], or heart 
transplantation [15] during the study period were excluded. Finally, patients who received a non-STEMI diagnosis were excluded. Figure 1 illustrates the patient selection process.

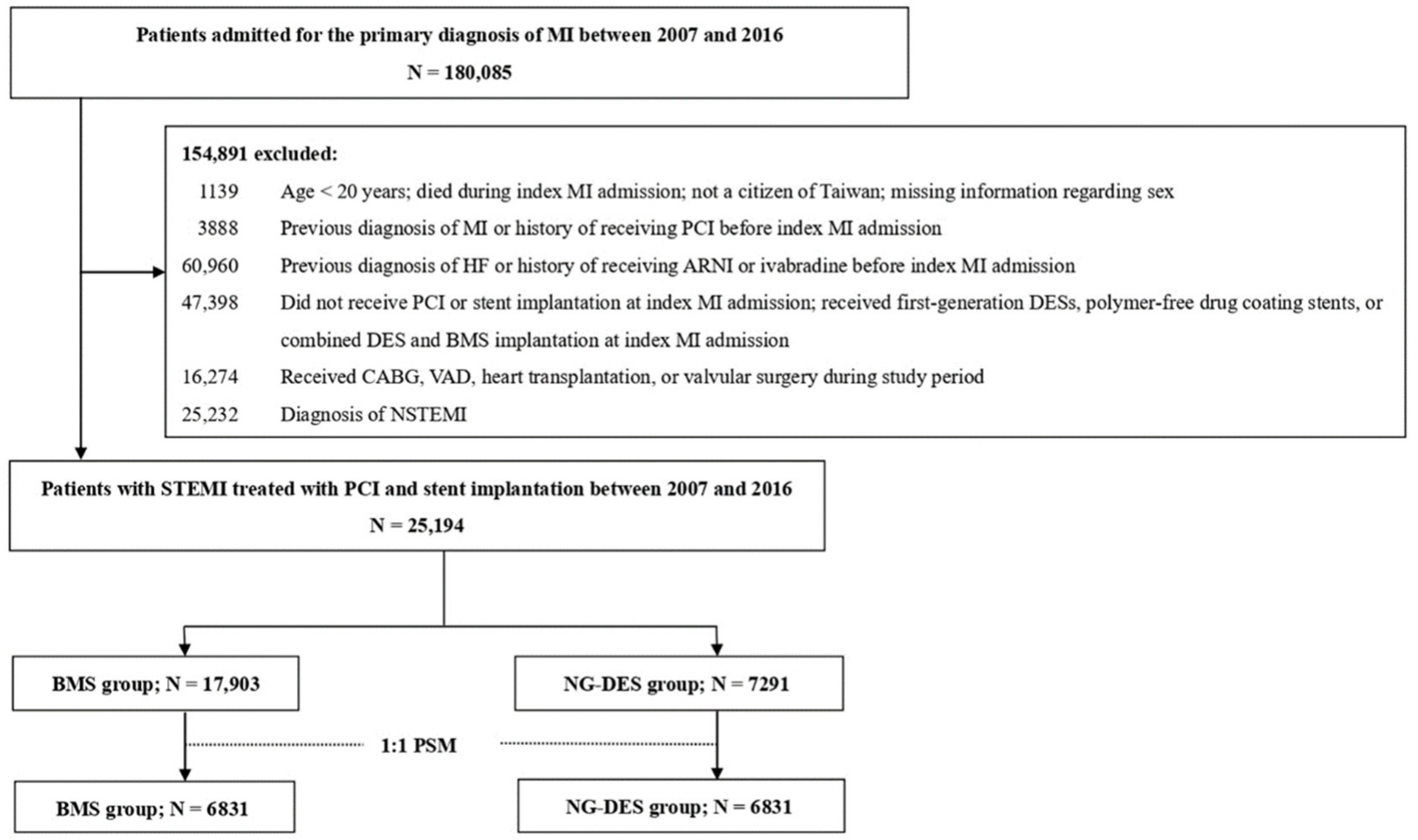

Figure 1. Patient selection process. ARNI-angiotensin receptor/neprilysin inhibitor; BMS-baremetal stent; CABG—coronary artery bypass grafting; DES — drug-eluting stent; HF-heart failure; $\mathrm{MI}$ = myocardial infarction; N-number; NG-DES—new-generation drug-eluting stent; NSTEMInon-ST-elevation myocardial infarction; PCI-percutaneous coronary intervention; PSM-propensity score matching; STEMI-ST-elevation myocardial infarction; VAD—ventricular assist device.

\subsection{Propensity Score Matching}

To reduce potential bias in patient selection, we used propensity score matching (PSM) to select pairs of patients with similar baseline characteristics but different implanted stent types (i.e., NG-DES or BMS). PSM is being increasingly used in studies estimating the treatment effects by employing observational data [16]. In the present study, pairs of patients were matched on the basis of the logit of the propensity score by using calipers of widths equal to 0.2 of the standard deviation.

Covariates used to calculate the propensity score are listed in Table 1: age, sex, index MI admission year, comorbidities, prescribed medications, multivessel PCI use, intraaortic balloon pump (IABP) use, $\mathrm{CHA}_{2} \mathrm{DS}_{2}$-VASc score (including congestive $\mathrm{HF}$, hypertension, age $\geq 75$ years, diabetes, stroke/transient ischemic attack, vascular disease, age $=65-74$ years, female sex) [17], ORBIT score (including age $\geq 75$ years, insufficient kidney function, treatment with any antiplatelet, a positive clinical history of bleeding, and anemia or abnormal hemoglobin) [18], and Academic Research Consortium (ARC)-defined major (including history of malignancy, use of anticoagulants, end-stage chronic kidney disease, and intracranial hemorrhage) and minor (including age $\geq 75$ years, history of ischemic stroke, and history of bleeding events requiring hospitalization or transfusion) bleeding risk criteria [19]. 
Table 1. Baseline characteristics of patients with STEMI receiving NG-DES or BMS before and after PSM.

\begin{tabular}{|c|c|c|c|c|c|c|c|c|c|c|}
\hline & \multicolumn{5}{|c|}{ Before PSM } & \multicolumn{5}{|c|}{ After PSM } \\
\hline & \multicolumn{2}{|c|}{ NG-DES } & \multicolumn{2}{|c|}{ BMS } & \multirow[t]{2}{*}{ SMD * } & \multicolumn{2}{|c|}{ NG-DES } & \multicolumn{2}{|c|}{ BMS } & \multirow[t]{2}{*}{ SMD * } \\
\hline & $n$ & $(\%)$ & $n$ & $(\%)$ & & $n$ & $(\%)$ & $n$ & $(\%)$ & \\
\hline $\mathrm{N}(\%)$ & 7291 & & 17,903 & & & 6831 & & 6831 & & \\
\hline Age, mean (SD) & \multicolumn{2}{|c|}{$59.0(12.5)$} & \multicolumn{2}{|c|}{$60.5(13.4)$} & & \multicolumn{2}{|c|}{$59.2(12.4)$} & \multicolumn{2}{|c|}{$59.2(12.4)$} & \\
\hline \multicolumn{11}{|l|}{ Age group, $n(\%)$} \\
\hline $20-44$ & 873 & $(12.0)$ & 1993 & (11.1) & 0.026 & 775 & (11.3) & 780 & (11.4) & 0.002 \\
\hline $45-64$ & 4144 & $(56.8)$ & 9354 & $(52.2)$ & 0.092 & 3881 & $(56.8)$ & 3881 & $(56.8)$ & $<0.001$ \\
\hline $65-74$ & 1341 & $(18.4)$ & 3399 & $(19.0)$ & 0.015 & 1287 & $(18.8)$ & 1282 & (18.8) & 0.002 \\
\hline$\geq 75$ & 933 & $(12.8)$ & 3157 & $(17.6)$ & 0.135 & 888 & (13.0) & 888 & $(13.0)$ & $<0.001$ \\
\hline Male (\%) & 8935 & (81.4) & 15,086 & (84.3) & 0.069 & 5989 & (87.7) & 5989 & $(87.7)$ & $<0.001$ \\
\hline \multicolumn{11}{|l|}{ Clinical data of index PCI } \\
\hline \multicolumn{11}{|l|}{ Diagnostic year } \\
\hline 2007-2010 & 873 & $(12.0)$ & 7504 & $(41.9)$ & 0.717 & 868 & $(12.7)$ & 868 & (12.7) & $<0.001$ \\
\hline 2011-2013 & 2334 & $(32.0)$ & 5567 & $(31.1)$ & 0.020 & 2279 & $(33.4)$ & 2279 & (33.4) & $<0.001$ \\
\hline 2014-2016 & 4084 & $(56.0)$ & 4832 & $(27.0)$ & 0.616 & 3684 & $(53.9)$ & 3684 & (53.9) & $<0.001$ \\
\hline Multivessel PCI & 846 & $(11.6)$ & 1661 & $(9.3)$ & 0.076 & 715 & $(10.5)$ & 586 & (8.6) & 0.064 \\
\hline Number of stents, mean (SD) & \multicolumn{2}{|c|}{$1.23(0.53)$} & \multicolumn{2}{|c|}{$1.28(0.57)$} & & \multicolumn{2}{|c|}{$1.23(0.53)$} & \multicolumn{2}{|c|}{$1.23(0.52)$} & \\
\hline IABP use, yes, $n(\%)$ & 368 & $(5.3)$ & 1761 & $(9.8)$ & 0.172 & 375 & $(5.5)$ & 364 & $(5.3)$ & 0.007 \\
\hline \multicolumn{11}{|l|}{ Comorbidity } \\
\hline $\mathrm{DM}$ & 2122 & $(29.1)$ & 5537 & $(30.9)$ & 0.040 & 2006 & $(29.4)$ & 1988 & $(29.1)$ & 0.006 \\
\hline HTN & 3921 & $(53.8)$ & 9326 & $(52.1)$ & 0.034 & 3655 & (53.5) & 3636 & $(53.2)$ & 0.006 \\
\hline Dyslipidemia & 3784 & $(51.9)$ & 7927 & $(44.3)$ & 0.153 & 3523 & $(51.6)$ & 3440 & $(50.4)$ & 0.024 \\
\hline CVD & 373 & $(5.1)$ & 1391 & $(7.8)$ & 0.108 & 362 & (5.3) & 351 & $(5.1)$ & 0.007 \\
\hline $\mathrm{AF}$ & 217 & (3.0) & 593 & (3.3) & 0.019 & 201 & $(2.9)$ & 206 & (3.0) & 0.004 \\
\hline COPD/asthma & 306 & $(4.2)$ & 962 & $(5.4)$ & 0.055 & 298 & $(4.4)$ & 284 & $(4.2)$ & 0.010 \\
\hline Dementia/parkinsonism & 76 & $(1.0)$ & 359 & $(2.0)$ & 0.079 & 72 & $(1.1)$ & 72 & $(1.1)$ & $<0.001$ \\
\hline OA/RA/rheumatism & 697 & (9.6) & 1906 & $(10.6)$ & 0.036 & 656 & (9.6) & 656 & (9.6) & $<0.001$ \\
\hline \multicolumn{11}{|l|}{$\mathrm{CHA}_{2} \mathrm{DS}_{2}$-VASc score } \\
\hline$\geq 2$ & 5478 & $(75.1)$ & 13,771 & $(76.9)$ & 0.042 & 5121 & $(75.0)$ & 5115 & $(74.9)$ & 0.002 \\
\hline $0-1$ & 1813 & $(24.9)$ & 4132 & $(23.1)$ & 0.042 & 1710 & $(25.0)$ & 1716 & $(25.1)$ & 0.002 \\
\hline Medication use & & & & & & & & & & \\
\hline ACEI/ARB & 5876 & $(80.6)$ & 14,128 & $(78.9)$ & 0.042 & 5510 & $(80.7)$ & 5476 & $(80.2)$ & 0.013 \\
\hline Beta-blockers & 5661 & $(77.6)$ & 12,623 & $(70.5)$ & 0.163 & 5240 & $(76.7)$ & 5203 & $(76.2)$ & 0.013 \\
\hline Nitrates & 67,32 & (92.3) & 16,168 & $(90.3)$ & 0.072 & 6297 & $(92.2)$ & 6174 & $(90.4)$ & 0.064 \\
\hline Aspirin & 7243 & (99.3) & 17,695 & $(98.8)$ & 0.053 & 6784 & (99.3) & 6792 & (99.4) & 0.015 \\
\hline P2Y12 inhibitors & 7271 & (99.7) & 17,814 & (99.5) & 0.036 & 6811 & (99.7) & 6818 & $(99.8)$ & 0.021 \\
\hline Statins & 6271 & $(86.0)$ & 13,028 & $(72.8)$ & 0.332 & 5840 & (85.5) & 5861 & $(85.8)$ & 0.009 \\
\hline PPIs & 755 & $(10.4)$ & 2501 & $(14.0)$ & 0.111 & 709 & $(10.4)$ & 750 & $(11.0)$ & 0.019 \\
\hline Steroids & 687 & $(9.4)$ & 2414 & $(13.5)$ & 0.128 & 667 & $(9.8)$ & 701 & $(10.3)$ & 0.017 \\
\hline NSAIDs & 2000 & $(27.4)$ & 6543 & $(36.5)$ & 0.196 & 1940 & $(28.4)$ & 1784 & $(26.1)$ & 0.051 \\
\hline ORBIT score & & & & & & & & & & \\
\hline$\geq 3$ & 179 & $(2.5)$ & 821 & $(4.6)$ & 0.116 & 173 & (2.5) & 182 & $(2.7)$ & 0.008 \\
\hline $0-2$ & 7112 & $(97.5)$ & 17,082 & $(95.4)$ & 0.116 & 6658 & $(97.5)$ & 6649 & (97.3) & 0.008 \\
\hline ARC criteria of bleeding risk & & & & & & & & & & \\
\hline
\end{tabular}


Table 1. Cont.

\begin{tabular}{|c|c|c|c|c|c|c|c|c|c|c|}
\hline & \multicolumn{5}{|c|}{ Before PSM } & \multicolumn{5}{|c|}{ After PSM } \\
\hline & \multicolumn{2}{|c|}{ NG-DES } & \multicolumn{2}{|c|}{ BMS } & \multirow[t]{2}{*}{ SMD * } & \multicolumn{2}{|c|}{ NG-DES } & \multicolumn{2}{|c|}{ BMS } & \multirow[t]{2}{*}{ SMD * } \\
\hline & $n$ & $(\%)$ & $n$ & $(\%)$ & & $n$ & $(\%)$ & $n$ & $(\%)$ & \\
\hline \multicolumn{11}{|l|}{ Major criteria, yes } \\
\hline Malignancy & 183 & $(2.5)$ & 513 & $(2.9)$ & 0.022 & 172 & $(2.5)$ & 181 & $(2.6)$ & 0.008 \\
\hline $\begin{array}{l}\text { Long-term use of } \\
\text { anticoagulants }\end{array}$ & 98 & $(1.3)$ & 354 & $(2.0)$ & 0.050 & 95 & $(1.4)$ & 100 & $(1.5)$ & 0.006 \\
\hline End-stage CKD & 15 & $(0.2)$ & 55 & $(0.3)$ & 0.020 & 15 & $(0.2)$ & 19 & $(0.3)$ & 0.012 \\
\hline $\mathrm{ICH}$ & 36 & $(0.5)$ & 150 & $(0.8)$ & 0.042 & 34 & $(0.5)$ & 38 & $(0.6)$ & 0.008 \\
\hline \multicolumn{11}{|l|}{ Minor criteria, yes } \\
\hline Age $\geq 75$ & 933 & $(12.8)$ & 3157 & $(17.6)$ & 0.135 & 888 & $(13.0)$ & 888 & (13.0) & $<0.001$ \\
\hline Ischemic stroke & 207 & $(2.8)$ & 796 & $(4.4)$ & 0.086 & 198 & $(2.9)$ & 201 & $(2.9)$ & 0.003 \\
\hline $\begin{array}{c}\text { Bleeding events requiring } \\
\text { hospitalization or transfusion }\end{array}$ & 90 & $(1.2)$ & 444 & $(2.5)$ & 0.092 & 88 & $(1.3)$ & 103 & $(1.5)$ & 0.019 \\
\hline \multicolumn{11}{|l|}{ DAPT at discharge of index MI } \\
\hline Aspirin & 7228 & $(99.1)$ & 17,654 & (98.6) & 0.050 & 6770 & (99.1) & 6779 & (99.2) & 0.015 \\
\hline P2Y12 inhibitors & 7271 & (99.7) & 17,811 & (99.5) & 0.038 & 6811 & $(99.7)$ & 6818 & (99.8) & 0.021 \\
\hline
\end{tabular}

ACEI-angiotensin-converting enzyme inhibitor; ARB - angiotensin II receptor blocker; ARC-Academic Research Consortium; AF-atrial fibrillation; BMS-bare-metal stent; $\mathrm{CHA}_{2} \mathrm{DS}_{2}$-VASc score-congestive heart failure, hypertension, age $\geq 75$ years, diabetes, stroke/transient ischemic attack, vascular disease, age 65 to 74 years, female sex; CKD—chronic kidney disease; COPD—chronic obstructive pulmonary disease; CVDcerebrovascular disease; DES—drug-eluting stent; DM-diabetes mellitus; HHF-hospitalization for heart failure; HTN_hypertension; IABP_intraaortic balloon pump; ICH—intracranial hemorrhage; NG-DES—newergeneration drug-eluting stent; NSAIDs—nonsteroidal anti-inflammatory drugs; OA—osteoarthritis; OACs—oral anticoagulants; ORBIT score-age $\geq 75$ years, bleeding history, chronic kidney disease, treatment with antiplatelet; $\mathrm{PCI}$ - percutaneous coronary intervention; PPIs—-proton pump inhibitors; PSM = propensity score matching; RA—rheumatoid arthritis; SD—standard deviation; SMD—standardized mean difference; STEMI-ST-elevation myocardial infarction. *-difference in means or proportions divided by standard error and imbalance defined as an absolute value $>0.1$.

Comorbidities were defined if patients had two or more diagnostic claims within 1 year prior to the index MI admission. Prescribed medications included angiotensinconverting enzyme inhibitors (ACEIs) or angiotensin II receptor blockers (ARBs), betablockers, nitrates, aspirin, $\mathrm{P}_{2} \mathrm{Y}_{12}$ inhibitors, statins, proton pump inhibitors (PPIs), steroids, and nonsteroidal anti-inflammatory drugs (NSAIDs). The diagnostic codes for diseases and the ATC codes for medications are listed in Table S1.

We used multivessel PCI and $\mathrm{CHA}_{2} \mathrm{DS}_{2}$-VASc scores as indicators to represent the severity of coronary artery disease (CAD) [20]. To consider the potential risk of bleeding complications, we referred to patients who had at least one major or two minor ARC criteria for bleeding risk as patients with ARC-HBR according to the ARC's definition [19]. We also used the ORBIT score to assist the assessment of patients' bleeding risk, in which patients with ORBIT scores of 0-2 were classified as low risk, whereas those with ORBIT scores of $\geq 3$ were classified as medium to high risk [18].

\subsection{Study Outcomes}

The main study outcome was the occurrence of HHF (ICD-9-CM code 428 and ICD-10 code I50) after stent implantation. The study patients were followed up for 5 years. Those who died before developing HF during the follow-up period were considered to have competing risks.

\subsection{Statistical Methods}

Primary analysis was performed to examine the risk of HHF associated with the use of the two stent types (i.e., NG-DES and BMS) in patients with STEMI. The consistency of the treatment effect was evaluated among eight prespecified subgroups stratified by 
age, sex, number of treated vessels, number of implanted stents, use of an IABP, $\mathrm{CHA}_{2} \mathrm{DS}_{2}-$ VASc scores, ORBIT scores, and whether the patients were at ARC-HBR [19]. We used a competing risk model in which death was considered a competing risk and reported the adjusted subdistribution hazard ratio (SHR) to account for the possibility that patients might have died before HHF occurrence. The variables listed in Table 1 were used in the competing risk model when examining the association between implanted stent types and the occurrence of HHF. To further examine the abovementioned association in patients with STEMI who were compliant with guideline-recommended duration of DAPT [2,3], we also performed a separate analysis of patients who received DAPT for an undefined duration of $>6$ months after the index MI admission. All analyses were performed using SAS/STAT 9.4 (SAS Institute, Cary, NC, USA) and STATA 14 software (Stata Corp, College Station, TX, USA). A standardized mean difference (SMD) $>0.1$ indicated imbalance between the two groups, and a $p$ of $<0.05$ indicated a significant association between risk of HHF and type of stent used.

\section{Results}

Of the 25,194 patients with STEMI who received PCI and stent implantation included in this study, 7291 (28.9\%) received NG-DES implantation. The patients who were aged $\geq 75$ years had less frequently received NG-DES implantation than those patients who were $<75$ years. Overall, the use of NG-DESs gradually increased over time. Compared with patients in the BMS group, those in the NG-DES group had a higher prevalence of dyslipidemia; had a lower prevalence of cerebrovascular disease; had more frequently used beta-blockers and statins; had less frequently used PPIs, steroids, and NSAIDs; had less frequently used IABP; and had lower ORBIT scores (Table 1). Using PSM, we selected 6831 patient pairs, and the baseline characteristics in the NG-DES and BMS groups were highly similar (Table 1).

The rates of cumulative incidence competing risk (CICR) of HHF after stent implantation at the 1, 2, 3, 4, and 5 year follow-up were lower in the NG-DES group (3.79\%, $5.21 \%, 6.15 \%, 7.01 \%$, and $8.29 \%$, respectively) than in the BMS group $(4.51 \%, 6.21 \%, 7.32 \%$, $8.33 \%$, and $9.83 \%$, respectively; Figure 2). After adjustments for covariates listed in Table 1, NG-DES implantation in patients with STEMI was associated with a decreased HHF risk at the 5 year follow-up compared with BMS implantation (adjusted SHR 0.82, 95\% confidence interval (CI) 0.72-0.92, $p=0.001$; Figure 2). We also conducted an analysis based on types of NG-DESs categorized by their coated drugs (Table S2) and observed that the rates of CICR and the risk of stent-associated HHF were comparable among different NG-DESs.

The subgroup analysis showed that the benefits of NG-DESs over BMSs were consistent in the following prespecified groups: patients who received two or more stents versus those who received one stent and patients with a $\mathrm{CHA}_{2} \mathrm{DS}_{2}$-VASc score of $\geq 2$ versus those with a $\mathrm{CHA}_{2} \mathrm{DS}_{2}$-VASc score of $<2$ (Figure 3). Meanwhile, patients with STEMI who were aged $<75$ years, male, and received PCI for two or more vessels did not use IABP, had ORBIT scores $<3$, and were not at ARC-HBR received more benefits from NG-DESs than did their counterparts (Figure 3 ).

After excluding patients who had received $<6$ months of DAPT, received no DAPT, died, or had a diagnosis of HF within 6 months after the index MI, we generated a second cohort comprising those who had received DAPT for $>6$ months after stent implantation (Figure S1). Baseline characteristics of the NG-DES and BMS groups in the second cohort were similar after using PSM (Table S3). Of the patients who received DAPT for $>6$ months after stent implantation, the CICR of HHF at 1, 2, 3, 4, and 5 year follow-ups remained lower in the NG-DES group $(1.81 \%, 2.96 \%, 3.86 \%, 4.94 \%$, and $6.02 \%$, respectively) than in the BMS group $(2.34 \%, 3.82 \%, 4.99 \%, 6.38 \%$, and $7.75 \%$, respectively; adjusted SHR 0.78 , $95 \%$ CI 0.62-0.97, $p=0.024$; Figure S2). In addition, the results of subgroup analysis in the second cohort were similar to those in the original cohort (Figure S3). 


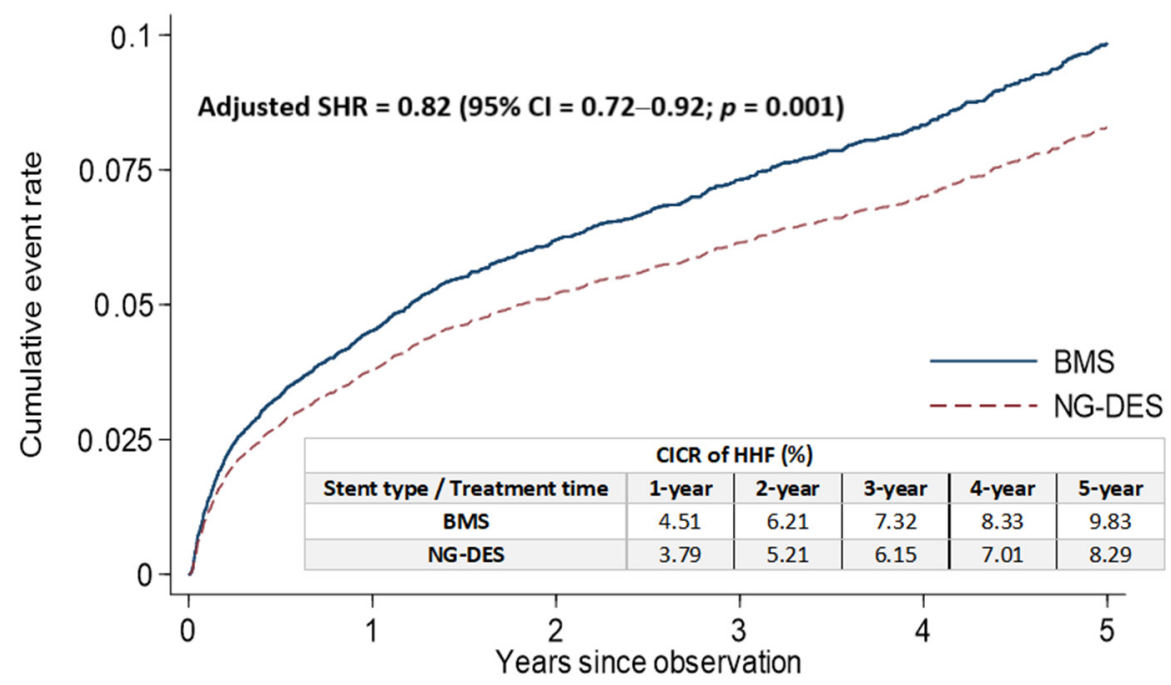

Figure 2. Cumulative incidence of HHF for competing risk among patients with STEMI receiving NG-DES or BMS implantation. BMS—bare-metal stent; CI—confidence interval; CICR-cumulative incidence competing risk; HHF_-hospitalization for heart failure; NG-DES—new-generation drugeluting stent; SHR—subdistribution hazard ratio; STEMI—ST-elevation myocardial infarction.

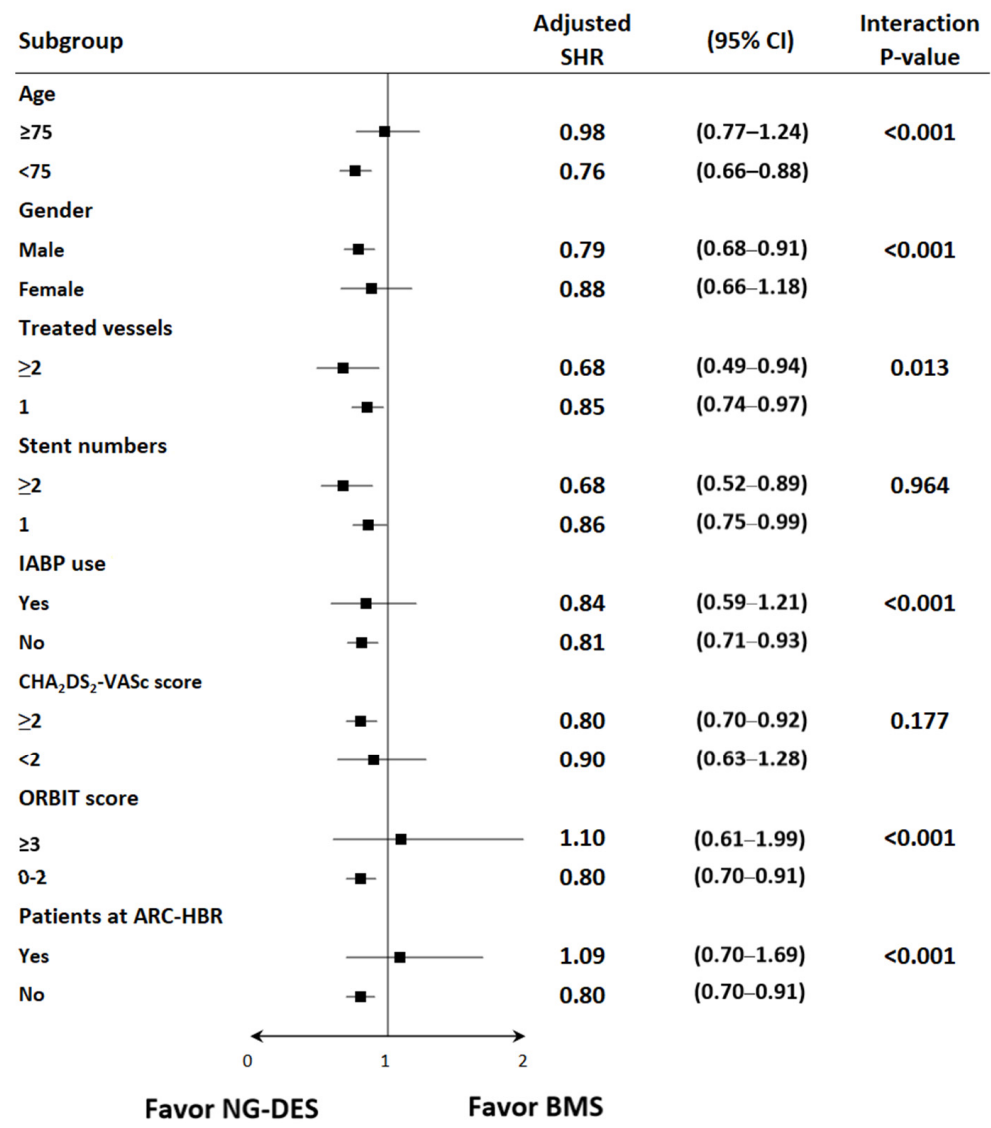

Figure 3. Subgroup analysis of the risk of HHF among patients with STEMI receiving NG-DESs (reference group) and BMSs. Adjusted SHR was adjusted for baseline covariates, including age, sex, year of index MI admission, comorbidities, prescribed medications, use of IABP during PCI, multivessel $\mathrm{PCI}, \mathrm{CHA}_{2} \mathrm{DS}_{2}$-VASc scores, ORBIT scores, and various ARC criteria for HBR. Abbreviations are defined in the footnote of Table 1. 


\section{Discussion}

In our knowledge, the present study is the first real-world evidence using a large-scale, population-based database to demonstrate that NG-DES use, compared with BMS use, is associated with a reduced risk of HHF among patients with STEMI at the 5 year followup. The Harmonizing Outcomes with Revascularization and Stents in Acute Myocardial Infarction (HORIZONS-AMI) trial has reported that HF incidence rate in patients with STEMI was $5.2 \%$ at the 2 year follow-up [21]. However, the HORIZONS-AMI trial reported a relative short-term observation in patients who received specific types of first-generation DES and BMS [22], thereby hardly inferring the long-term effect of NG-DES implantation on the risk of HHF in patients with STEMI. Our present study demonstrated not only a long-term benefit of NG-DES in reducing the risk of HHF but also a decreased risk of repeat PCI (Table S4) and recurrent MI (Table S5) associated with NG-DES implantation, which may limit adverse myocardial remodeling and explain our findings.

Although a 6 month duration of DAPT is considered in patients with STEMI who exhibit HBR features, irrespective of the implanted stent type $[2,3,23]$, the choice of stent types for patients with STEMI deemed to have HBR is challenging because of the lack of consensus-based definition of HBR and the patients' uncertain compliance with DAPT [24]. A recent meta-analysis [24] analyzed three landmark RCTs [25-27] and determined that NG-DES with 1 month DAPT in patients with various high-risk features of bleeding complications lowered the risk of adverse events compared with BMS implantation. However, only a small portion of patients (4-15\%) was identified as having STEMI in these aforementioned RCTs [25-27]. In addition, the criteria to define HBR used in each trial were quite different [25-27], which resulted in poor generalizability to real-world scenarios. In this study, we did not observe the superiority of NG-DES over BMS when the analyses focused on patients with STEMI who were at ARC-HBR identified by the ARC's definition. Our results were similar to those of patients with STEMI deemed to have various high-risk features of bleeding complications in the previous study [28]. Patients who were at ARC-HBR in our study cohort were older in age and had more comorbidities compared with those who were not at ARC-HBR (Table S6), which might lead to a higher risk of developing HF and counteract the benefits of NG-DES. Further RCTs using a consensus-based definition of HBR are needed to determine which type of stent is favored in patients with STEMI who are at HBR.

Approximately 50\% of patients with STEMI have multivessel disease in which the current guidelines have recommended that PCI for non-infarct-related arteries can be considered in selected patients [2,29]. Recently, the Complete Revascularization with Multivessel PCI for Myocardial Infarction (COMPLETE) trial [30] showed that complete revascularization with multivessel PCI was superior to IRA-only PCI in reducing adverse cardiovascular outcomes among patients with STEMI and multivessel disease. However, the COMPLETE trial failed to demonstrate which type of stents was favored in multivessel PCI strategy. The data presented here could provide further implication for considering NG-DESs to improve clinical outcomes in patients with STEMI who received multivessel PCI strategy and multiple stent implantation.

In the subgroup analysis, we did not observe the superiority of NG-DES over BMS in older and female patients. Female patients with STEMI tend to have an increased extent of endothelial inflammation and microvascular dysfunction and have more baseline risk profiles, such as diabetes, hypertension, obesity, and psychological distress, which may influence the risk of HF to a larger degree compared with male patients with STEMI [31]. In addition, elderly patients may frequently have contraindications or intolerance to ACEIs, ARBs, and beta-blockers that are guideline-directed medications for HF [10]. These aforementioned differences may contribute to sex and age disparities in the benefits of NG-DES.

The strength of this study is the use of large-scale real-world data to investigate a prominent concern that is hardly addressed using RCTs or meta-analyses in the contemporary era. We also used PSM to reduce the potential patient selection bias. Moreover, in our analyses, we considered various high-risk characteristics of bleeding complications, includ- 
ing the ORBIT scores and ARC criteria for HBR, which were rarely included in previous studies, so that we could observe the influence of HBR features on stent-associated HF risk in patients with STEMI who received undefined duration of DAPT.

This study has some limitations. First, even when PSM is applied, a nonrandomized retrospective design may introduce bias because of some confounders, such as patients baseline left ventricular ejection fraction and serum brain natriuretic peptide levels, angiographic findings of $\mathrm{PCI}$, and the extent of patients' CAD that could not be measured in the claim-based data. Moreover, the NHIRD did not provide certain clinical information, such as stent thrombosis and TVF, that might influence the occurrences of HHF. In the present study, we showed that the use of NG-DES was superior to BMS in terms of a reduced risk of repeat PCI (Table S4) and recurrent MI (Table S5), which might be two proxy indicators of stent thrombosis and TVF. A long period of observation in our present study, which included heterogeneity of patient composition and treatment methods, might also have introduced some bias in our results. Second, the implementation of PSM undoubtedly reduced the external validity of our study because only a subset of treated patients was analyzed; however, despite its limitations, PSM remains an acceptable approach that has been validated in RCTs [32]. Finally, the study cohort was limited to an Asian population; therefore, the results might not be applicable to other populations. Future prospective randomized studies are warranted to confirm our findings.

\section{Conclusions}

The NG-DES implantation exhibited a superiority over BMS in reducing the 5 year risk of HHF among patients with STEMI who received undefined duration of DAPT; the results are consistent with those for patients who had received DAPT for $>6$ months. Overall, these real-world data suggest that NG-DESs significantly prevent HHF after PCI in patients with STEMI.

Supplementary Materials: The following supporting information can be downloaded at https: //www.mdpi.com/article/10.3390/jpm12030369/s1. Table S1: Disease diagnostic coding and ATC code for medication. Table S2: The rates of CICR and the risk of stent-associated HHF between different NG-DESs categorized by coated drugs. Figure S1: Selection process of patients with STEMI who received DAPT for an undefined duration of longer than 6 months following stent implantation. Table S3: Baseline characteristics of patients with STEMI who received DAPT for an undefined duration of longer than 6 months following stent implantation. Figure S2: Cumulative incidence of HHF for competing risk among patients with STEMI who received DAPT for an undefined duration of longer than 6 months following stent implantation. Figure S3: Subgroup analysis of the risk of HHF among patients with STEMI who received DAPT for an undefined duration of longer than 6 months following stent implantation. Table S4: The rates of CICR and the risk of repeat PCI in patients with STEMI receiving NG-DESs compared with those patients with STEMI receiving BMSs. Table S5: The rates of CICR and the risk of recurrent MI in patients with STEMI receiving NG-DESs compared with those patients with STEMI receiving BMSs. Table S6: Baseline characteristics of patients with STEMI who were at ARC-HBR and non-ARC-HBR after PSM.

Author Contributions: Conceptualization, L.-N.C. and C.-F.L.; methodology, L.-N.C., C.-C.C., Y.-H.C. and C.-F.L.; formal analysis, L.-N.C., F.-C.Y., C.-T.T. and H.-Y.L.; investigation, L.-N.C., H.-Y.L., H.-I.Y. and C.-F.L.; data curation, C.-C.C. and Y.-H.C.; writing-original draft preparation, L.-N.C. and C.-F.L.; writing-review and editing, L.-N.C., Y.-H.C. and C.-F.L.; visualization, L.-N.C., C.-C.C., Y.-H.C., F.-C.Y., C.-T.T., H.-Y.L., H.-I.Y. and C.-F.L.; supervision, C.-F.L. All authors have read and agreed to the published version of the manuscript.

Funding: This research received no external funding.

Institutional Review Board Statement: The study was conducted in accordance with the Declaration of Helsinki and approved by the Institutional Review Board of MacKay Memorial Hospital (MMHIRB; approval no. 20MMHIS351e).

Informed Consent Statement: Patient consent was waived under the full review process of the MMHIRB because individual identifiers in the NHIRD have been encrypted and cannot be recognized. 
Data Availability Statement: The datasets used and/or analyzed during the current study are available from the corresponding author on reasonable request.

Acknowledgments: This study was supported by the Health and Clinical Research Data Center, Office of Data, Taipei Medical University.

Conflicts of Interest: The authors declare no conflict of interest.

\section{References}

1. Gerber, Y.; Weston, S.A.; Enriquez-Sarano, M.; Berardi, C.; Chamberlain, A.M.; Manemann, S.M.; Jiang, R.; Dunlay, S.M.; Roger, V.L. Mortality Associated With Heart Failure After Myocardial Infarction: A Contemporary Community Perspective. Circ. Heart Fail. 2016, 9, e002460. [CrossRef] [PubMed]

2. Ibanez, B.; James, S.; Agewall, S.; Antunes, M.J.; Bucciarelli-Ducci, C.; Bueno, H.; Caforio, A.L.P.; Crea, F.; Goudevenos, J.A.; Halvorsen, S.; et al. 2017 ESC Guidelines for the management of acute myocardial infarction in patients presenting with STsegment elevation: The Task Force for the management of acute myocardial infarction in patients presenting with ST-segment elevation of the European Society of Cardiology (ESC). Eur. Heart J. 2018, 39, 119-177. [PubMed]

3. Levine, G.N.; Bates, E.R.; Bittl, J.A.; Brindis, R.G.; Fihn, S.D.; Fleisher, L.A.; Granger, C.B.; Lange, R.A.; Mack, M.J.; Mauri, L.; et al. 2016 ACC/AHA Guideline Focused Update on Duration of Dual Antiplatelet Therapy in Patients With Coronary Artery Disease: A Report of the American College of Cardiology/American Heart Association Task Force on Clinical Practice Guidelines: An Update of the 2011 ACCF/AHA/SCAI Guideline for Percutaneous Coronary Intervention, 2011 ACCF/AHA Guideline for Coronary Artery Bypass Graft Surgery, 2012 ACC/AHA/ACP/AATS/PCNA/SCAI/STS Guideline for the Diagnosis and Management of Patients With Stable Ischemic Heart Disease, 2013 ACCF/AHA Guideline for the Management of ST-Elevation Myocardial Infarction, 2014 AHA/ACC Guideline for the Management of Patients With Non-ST-Elevation Acute Coronary Syndromes, and 2014 ACC/AHA Guideline on Perioperative Cardiovascular Evaluation and Management of Patients Undergoing Noncardiac Surgery. Circulation 2016, 134, e123-e155. [PubMed]

4. Räber, L.; Kelbæk, H.; Ostojic, M.; Baumbach, A.; Heg, D.; Tüller, D.; von Birgelen, C.; Roffi, M.; Moschovitis, A.; Khattab, A.A.; et al. Effect of biolimus-eluting stents with biodegradable polymer vs bare-metal stents on cardiovascular events among patients with acute myocardial infarction: The COMFORTABLE AMI randomized trial. JAMA 2012, 308, 777-787. [CrossRef] [PubMed]

5. Sabate, M.; Cequier, A.; Iñiguez, A.; Serra, A.; Hernandez-Antolin, R.; Mainar, V.; Valgimigli, M.; Tespili, M.; den Heijer, P.; Bethencourt, A.; et al. Everolimus-eluting stent versus bare-metal stent in ST-segment elevation myocardial infarction (EXAMINATION): 1 year results of a randomised controlled trial. Lancet 2012, 380, 1482-1490. [CrossRef]

6. Sabaté, M.; Brugaletta, S.; Cequier, A.; Iñiguez, A.; Serra, A.; Jiménez-Quevedo, P.; Mainar, V.; Campo, G.; Tespili, M.; den Heijer, P.; et al. Clinical outcomes in patients with ST-segment elevation myocardial infarction treated with everolimus-eluting stents versus bare-metal stents (EXAMINATION): 5-year results of a randomised trial. Lancet 2016, 387, 357-366. [CrossRef]

7. Kastrati, A.; Dibra, A.; Spaulding, C.; Laarman, G.J.; Menichelli, M.; Valgimigli, M.; Di Lorenzo, E.; Kaiser, C.; Tierala, I.; Mehilli, J.; et al. Meta-analysis of randomized trials on drug-eluting stents vs. bare-metal stents in patients with acute myocardial infarction. Eur. Heart J. 2007, 28, 2706-2713. [CrossRef]

8. Palmerini, T.; Biondi-Zoccai, G.; Della Riva, D.; Mariani, A.; Sabaté, M.; Valgimigli, M.; Frati, G.; Kedhi, E.; Smits, P.C.; Kaiser, C.; et al. Clinical outcomes with drug-eluting and bare-metal stents in patients with ST-segment elevation myocardial infarction: Evidence from a comprehensive network meta-analysis. J. Am. Coll. Cardiol. 2013, 62, 496-504. [CrossRef]

9. National Health Insurance: Laws and Regulations. Available online: https:/ / www.nhi.gov.tw/english/Content_List.aspx?n=A7 354F4F704B6377\&topn=A7354F4F704B6377 (accessed on 5 June 2020).

10. Ponikowski, P.; Voors, A.A.; Anker, S.D.; Bueno, H.; Cleland, J.G.F.; Coats, A.J.S.; Falk, V.; González-Juanatey, J.R.; Harjola, V.P.; Jankowska, E.A.; et al. 2016 ESC Guidelines for the diagnosis and treatment of acute and chronic heart failure: The Task Force for the diagnosis and treatment of acute and chronic heart failure of the European Society of Cardiology (ESC)Developed with the special contribution of the Heart Failure Association (HFA) of the ESC. Eur. Heart J. 2016, 37, 2129-2200.

11. Camenzind, E.; Steg, P.G.; Wijns, W. Stent thrombosis late after implantation of first-generation drug-eluting stents: A cause for concern. Circulation 2007, 115, 1440-1455. [CrossRef]

12. Moreyra, A.E.; Deng, Y.; Wilson, A.C.; Cosgrove, N.M.; Kostis, W.J.; Kostis, J.B.; MIDAS 18 Study Group. Incidence and trends of heart failure admissions after coronary artery bypass grafting surgery. Eur. J. Heart Fail. 2013, 15, 46-53. [CrossRef] [PubMed]

13. Burke, M.A.; Givertz, M.M. Assessment and management of heart failure after left ventricular assist device implantation. Circulation 2014, 129, 1161-1166. [CrossRef] [PubMed]

14. Groves, P. Valve disease: Surgery of valve disease: Late results and late complications. Heart 2001, 86, 715-721. [CrossRef]

15. Ambrosi, P.; Kreitmann, B.; Riberi, A.; Lambert, M.; Pankert, M.; Habib, G. Chronic heart failure in heart transplant recipients: Presenting features and outcome. Arch. Cardiovasc. Dis. 2016, 109, 254-259. [CrossRef]

16. D'Agostino, R.B., Jr. Propensity scores in cardiovascular research. Circulation 2007, 115, 2340-2343. [CrossRef] [PubMed]

17. Lip, G.Y.; Nieuwlaat, R.; Pisters, R.; Lane, D.A.; Crijns, H.J. Refining clinical risk stratification for predicting stroke and thromboembolism in atrial fibrillation using a novel risk factor-based approach: The euro heart survey on atrial fibrillation. Chest 2010, 137, 263-272. [CrossRef] [PubMed] 
18. O'Brien, E.C.; Simon, D.N.; Thomas, L.E.; Hylek, E.M.; Gersh, B.J.; Ansell, J.E.; Kowey, P.R.; Mahaffey, K.W.; Chang, P.; Fonarow, G.C.; et al. The ORBIT bleeding score: A simple bedside score to assess bleeding risk in atrial fibrillation. Eur. Heart J. 2015, 36, 3258-3264. [CrossRef]

19. Urban, P.; Mehran, R.; Colleran, R.; Angiolillo, D.J.; Byrne, R.A.; Capodanno, D.; Cuisset, T.; Cutlip, D.; Eerdmans, P.; Eikelboom, J.; et al. Defining High Bleeding Risk in Patients Undergoing Percutaneous Coronary Intervention. Circulation 2019, 140, $240-261$. [CrossRef]

20. Scudiero, F.; Zocchi, C.; De Vito, E.; Tarantini, G.; Marcucci, R.; Valenti, R.; Migliorini, A.; Antoniucci, D.; Marchionni, N.; Parodi, G. Relationship between $\mathrm{CHA}_{2} \mathrm{DS}_{2}$-VASc score, coronary artery disease severity, residual platelet reactivity and long-term clinical outcomes in patients with acute coronary syndrome. Int. J. Cardiol. 2018, 262, 9-13. [CrossRef]

21. Kelly, D.J.; Gershlick, T.; Witzenbichler, B.; Guagliumi, G.; Fahy, M.; Dangas, G.; Mehran, R.; Stone, G.W. Incidence and predictors of heart failure following percutaneous coronary intervention in ST-segment elevation myocardial infarction: The HORIZONS-AMI trial. Am. Heart J. 2011, 162, 663-670. [CrossRef]

22. Stone, G.W.; Witzenbichler, B.; Guagliumi, G.; Peruga, J.Z.; Brodie, B.R.; Dudek, D.; Kornowski, R.; Hartmann, F.; Gersh, B.J.; Pocock, S.J.; et al. Bivalirudin during primary PCI in acute myocardial infarction. N. Eng. J. Med. 2008, 358, 2218-2230. [CrossRef] [PubMed]

23. Valgimigli, M.; Bueno, H.; Byrne, R.A.; Collet, J.P.; Costa, F.; Jeppsson, A.; Jüni, P.; Kastrati, A.; Kolh, P.; Mauri, L.; et al. 2017 ESC focused update on dual antiplatelet therapy in coronary artery disease developed in collaboration with EACTS: The Task Force for dual antiplatelet therapy in coronary artery disease of the European Society of Cardiology (ESC) and of the European Association for Cardio-Thoracic Surgery (EACTS). Eur. Heart J. 2018, 39, 213-260. [PubMed]

24. Shah, R.; Rao, S.V.; Latham, S.B.; Kandzari, D.E. Efficacy and Safety of Drug-Eluting Stents Optimized for Biocompatibility vs Bare-Metal Stents with a Single Month of Dual Antiplatelet Therapy: A Meta-analysis. JAMA Cardiol. 2018, 3, 1050-1059. [CrossRef]

25. Ariotti, S.; Adamo, M.; Costa, F.; Patialiakas, A.; Briguori, C.; Thury, A.; Colangelo, S.; Campo, G.; Tebaldi, M.; Ungi, I.; et al. Is Bare-Metal Stent Implantation Still Justifiable in High Bleeding Risk Patients Undergoing Percutaneous Coronary Intervention: A Pre-Specified Analysis From the ZEUS Trial. JACC Cardiovasc. Interv. 2016, 9, 426-436. [CrossRef]

26. Varenne, O.; Cook, S.; Sideris, G.; Kedev, S.; Cuisset, T.; Carrié, D.; Hovasse, T.; Garot, P.; El Mahmoud, R.; Spaulding, C.; et al. Drug-eluting stents in elderly patients with coronary artery disease (SENIOR): A randomised single-blind trial. Lancet 2018, 391, 41-50. [CrossRef]

27. Urban, P.; Meredith, I.T.; Abizaid, A.; Pocock, S.J.; Carrié, D.; Naber, C.; Lipiecki, J.; Richardt, G.; Iñiguez, A.; Brunel, P.; et al. Polymer-free Drug-Coated Coronary Stents in Patients at High Bleeding Risk. N. Eng. J. Med. 2015, 373, 2038-2047. [CrossRef]

28. Naber, C.K.; Urban, P.; Ong, P.J.; Valdes-Chavarri, M.; Abizaid, A.A.; Pocock, S.J.; Fabbiocchi, F.; Dubois, C.; Copt, S.; Greene, S.; et al. Biolimus-A9 polymer-free coated stent in high bleeding risk patients with acute coronary syndrome: A Leaders Free ACS sub-study. Eur. Heart J. 2017, 38, 961-969. [CrossRef] [PubMed]

29. Levine, G.N.; Bates, E.R.; Blankenship, J.C.; Bailey, S.R.; Bittl, J.A.; Cercek, B.; Chambers, C.E.; Ellis, S.G.; Guyton, R.A.; Hollenberg, S.M.; et al. 2015 ACC/AHA/SCAI Focused Update on Primary Percutaneous Coronary Intervention for Patients With STElevation Myocardial Infarction: An Update of the 2011 ACCF/AHA/SCAI Guideline for Percutaneous Coronary Intervention and the 2013 ACCF/AHA Guideline for the Management of ST-Elevation Myocardial Infarction: A Report of the American College of Cardiology/American Heart Association Task Force on Clinical Practice Guidelines and the Society for Cardiovascular Angiography and Interventions. Circulation 2016, 133, 1135-1147.

30. Mehta, S.R.; Wood, D.A.; Storey, R.F.; Mehran, R.; Bainey, K.R.; Nguyen, H.; Meeks, B.; Di Pasquale, G.; López-Sendón, J.; Faxon, D.P.; et al. Complete Revascularization with Multivessel PCI for Myocardial Infarction. N. Eng. J. Med. 2019, 381, $1411-1421$. [CrossRef]

31. Lam, C.S.P.; Arnott, C.; Beale, A.L.; Chandramouli, C.; Hilfiker-Kleiner, D.; Kaye, D.M.; Ky, B.; Santema, B.T.; Sliwa, K.; Voors, A.A. Sex differences in heart failure. Eur. Heart J. 2019, 40, 3859-3868c. [CrossRef]

32. Austin, P.C. The use of propensity score methods with survival or time-to-event outcomes: Reporting measures of effect similar to those used in randomized experiments. Stat. Med. 2014, 33, 1242-1258. [CrossRef] [PubMed] 